\title{
Hodgkin's lymphoma associated with myelofibrosis: A case report
}

\author{
RONG FU, HONG YU, YU-HONG WU, HUI LIU and ZONG-HONG SHAO \\ Department of Hematology, Tianjin Medical University General Hospital, Tianjin 300052, P.R. China
}

Received August 27, 2014; Accepted May 20, 2015

DOI: $10.3892 / 01.2015 .3438$

\begin{abstract}
In the present study, the case of a patient with nodular sclerosing Hodgkin's lymphoma (NSHL) presenting with diffuse fibrosis of the bone marrow (BM) was reported. A 30-year-old male complained of fever for 1 year, as well as lumbago, lymph node swelling and night sweats for 3 months. A biopsy of the lymph nodes established a diagnosis of NSHL. Aspiration of BM was a dry tap, and the BM biopsy demonstrated marked myelofibrosis with increased proliferation of reticulin fiber. Multiple skeletal lesions were detected in the patient's vertebra, pelvis, sternum and bilateral femur by magnetic resonance imaging and computed tomography. Following numerous courses of chemotherapy and radiotherapy, remission of the lymphoma was achieved. Subsequently, the BM aspiration became possible, and BM biopsy demonstrated a reduction in fibrosis.
\end{abstract}

\section{Introduction}

Hodgkin's lymphoma (HL) accounts for $~ 30 \%$ of all lymphomas, presenting with generalized lymphadenopathy, hematological abnormalities and B symptoms (including fever, weight loss and night sweats) (1). Due to the development of highly active chemotherapy and radiotherapy strategies, patients with HL have an excellent prognosis following frontline therapy, and the 5-year progression-free survival rate can be as high as $75-80 \%$ (2). Myelofibrosis is a chronic myeloproliferative neoplasm characterized by clonal proliferation of myeloid hematopoietic cells and intramedullary fibrosis (3). Clinical manifestations of myelofibrosis include cytopenias, profound splenomegaly, bone pain, night sweats, weight loss, and fatigue (4). Secondary MF (SMF) is often observed in a number of hematological malignancies, including acute megakaryoblastic leukemia (5), chronic myeloid leukemia (6) and hairy cell leukemia (7); however, SMF is rare in lymphoid neoplasms. The present study reported a case of reversible

Correspondence to: Miss Rong Fu or Mr. Zong-Hong Shao, Department of Haematology, Tianjin Medical University General Hospital, 154 Anshan Street, Heping, Tianjin 300052, P.R. China

E-mail: florai@sina.com

E-mail: shaozonghong@sina.com

Key words: Hodgkin's lymphoma, myelofibrosis
MF associated with Hodgkin's lymphoma (HL), which was resolved following remission of lymphoma. Subsequent to a thorough review of the literature using the PubMed database (http://www.ncbi.nlm.nih.gov/pubmed), 7 reported cases of SMF accompanied by HL were identified (Table I); however, no HL cases with SMF have been previously reported in China. Previously reported lymphomas associated with MF were of various histological types, including T-cell lymphoma (8-12), B-cell lymphoma (13) and HL (14-17).

\section{Case report}

A 30-year-old male presented to the Tianjin Medical University General Hospital (Tianjin, China) on October 10, 2012 with fever that had persisted for 1 year, night sweats and lumbago that had persisted for 3 months, and weight loss of $25 \mathrm{~kg}$ within 1 year. At the time of admission, the patient exhibited a high temperature of $40.2^{\circ} \mathrm{C}$, a mild degree of pallor and palpable surface lymph nodes. Marked cervical, supraclavicular, axillary and inguinal lymphadenopathy had developed, with rubbery lymph nodes reaching 2-3 cm in size. The patient presented mild hepatomegaly and tender pain in the section of the lumbar vertebrae; however, no splenomegaly, adenopathy or skin eruptions were observed. A complete blood cell count revealed a normal white blood cell count

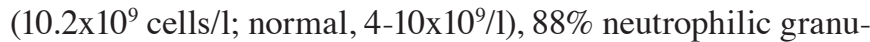
locytes and $10 \%$ lymphocytes, normocytic normochromic anemia (hemoglobin level, $81 \mathrm{~g} / \mathrm{l}$; normal, 120-160 g/l) and an

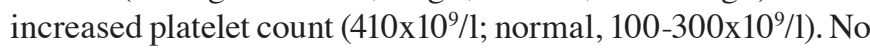
teardrop-shaped red blood cells or erythroblasts were detected in the peripheral blood. The prothrombin time and activated partial thromboplastin time were found to be $12.1 \mathrm{sec}$ and $37.1 \mathrm{sec}$ (normal, 20-40 sec), respectively, while the fibrinogen level was $511 \mathrm{mg} / \mathrm{dl}$ (normal, 1.8-40 mg/dl) and the D-dimer level was $497 \mu \mathrm{g} / \mathrm{l}$ (normal, 0-500 $\mu \mathrm{g} / \mathrm{l}$ ). Blood chemistry studies demonstrated elevated levels of lactate dehydrogenase (253 U/l; normal, 94-250 U/l) and $\beta_{2}$-microglobulin (1.41 mg/l; normal, 0.1-0.3 mg/l). The serum albumin level was $30 \mathrm{~g} / \mathrm{l}$ (normal, 35-55 g/l), and the hepatic and renal functions were normal. Direct and indirect Coombs tests were negative, while hypergammaglobulinemia and monoclonal gammopathy were not detected and blood sample cultures failed to reveal any pathogens. The patient was serologically negative for Epstein-Barr virus, hepatitis virus, cytomegalovirus, parvovirus $\mathrm{B} 19$, toxoplasmosis, coccidiomycosis, brucellosis and human immunodeficiency virus. In addition, the erythrocyte sedimentation rate was $64 \mathrm{~mm} / \mathrm{h}$ (normal, $0-15 \mathrm{~mm} / \mathrm{h}$ ), while 


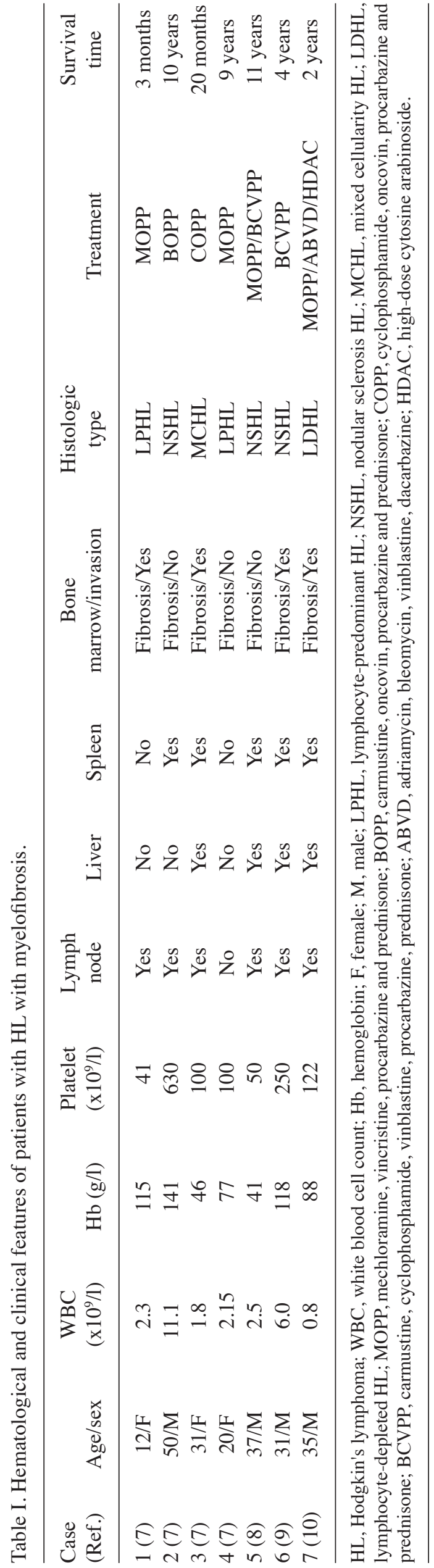

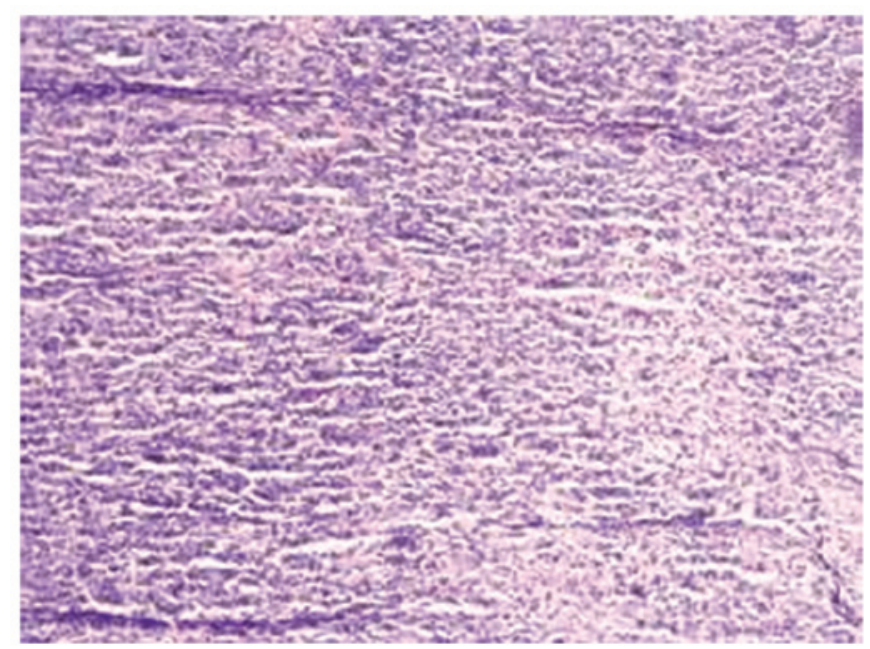

Figure 1. Microscopic view of the lymph node biopsy specimen, diagnosed with nodular sclerosing Hodgkin's lymphoma (magnification, x40; stain, hematoxylin and eosin).

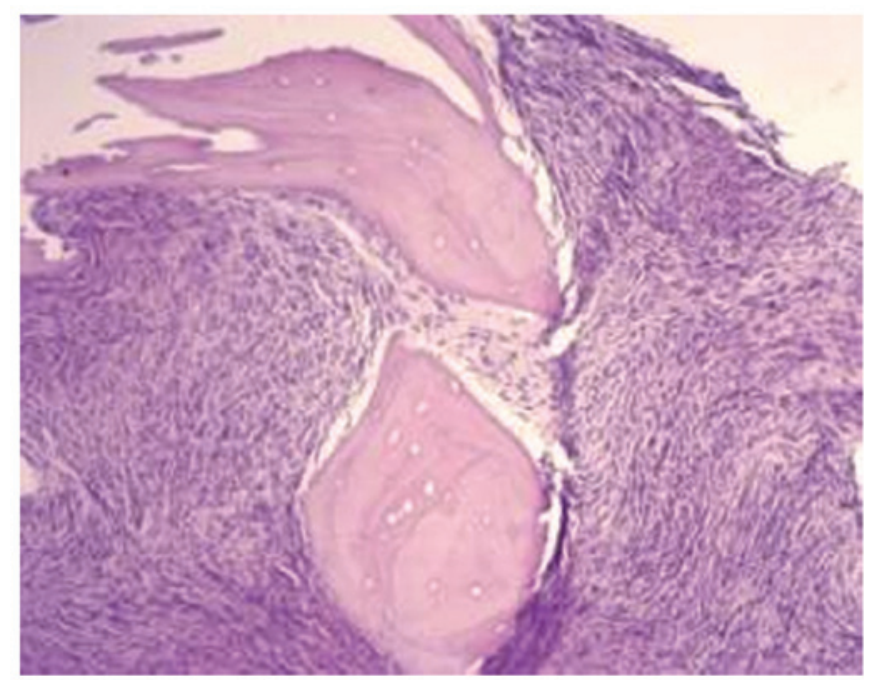

Figure 2. Microscopic view of the bone marrow biopsy specimen, diagnosed with myelofibrosis. Increased proliferation of fibroblasts and reticulin fiber is observed (magnification, $\mathrm{x} 40$; stain, hematoxylin and eosin).

immune screening, including autoantibody and complement, was negative. Finally, the CD4/CD8 ratio (1.01\%; normal, $0.8-2.5 \%$ ) was within the normal range.

A systemic computed tomography (CT) scan demonstrated multiple swellings of the bilateral cervical, supraclavicular, mediastinal, axillary, para-aortic, inguinal and mesenteric lymph nodes, an enlarged liver and no splenomegaly. A cranium CT scan demonstrated no lesions. A single-photon emission computed tomography bone scan revealed numerous areas of increased tracer uptake, indicating skeleton invasion. Fluorodeoxyglucose positron emission tomography was not performed at this time.

Biopsy of the cervical lymph node demonstrated disappearance of the normal architecture and neoplastic cells. Immunohistochemical staining demonstrated that the cells were positive for $\mathrm{CD} 30$, as well as for $\mathrm{CD} 20$ and $\mathrm{CD} 3$ in scattered cells, and negative for CD15. These findings were 
compatible with a diagnosis of NSHL (Fig. 1). Bone marrow (BM) aspirations of the sternum and iliac bone were dry taps, and biopsy of the BM from the iliac bone demonstrated diffuse MF, showing proliferation of fibroblasts and reticulin fiber, without an increase in megakaryocytes and without lymphomatous infiltration (Fig. 2).

Based on the aforementioned findings, the patient was diagnosed with an NSHL variant with MF. The lymphoma was classified as clinical stage IV (18). Subsequently, the patient underwent 4 courses of chemotherapy with ABVD (doxorubicin, $120 \mathrm{mg}$, days 1 and 15; bleomycin, $15 \mathrm{mg}$, days 1 and 15; vinblastine, $3 \mathrm{mg}$, days 1 and 15; and dacarbazine, $600 \mathrm{mg}$, days 1 and 15) achieving a partial response. The patient showed resolution of B symptoms with anemia improvement, and reduction in the size of the lymph nodes and liver. Aspiration of BM became possible and a BM biopsy demonstrated a hypocellular marrow without evidence of lymphoma and a reduction in fibrosis. Flow cytometric analyses of BM lymphocytes revealed that they were positive for CD7, CD3, CD5 and CD38, and negative for CD20, CD22, CD23, CD10, CD9, ZAP70 and CD79a. In addition, chromosome analysis of BM cells demonstrated a normal karyotype (46XY). A JAK2 mutation, V617F, was detected and immunoglobulin gene rearrangement was negative. However, small lymph nodes (1-2 cm in diameter) remained in the neck and supraclavicular after 4 courses of chemotherapy. Therefore, the patient was treated with 2 courses of BEACOPP (bleomycin, $15 \mathrm{mg}$, day 8; etoposide, $200 \mathrm{mg}$, days 1-3; doxorubicin, $80 \mathrm{mg}$, day 1; cyclophosphamide, 1,200 mg, day 1; vincristine, $4 \mathrm{mg}$, day 8; procarbazine, $700 \mathrm{mg}$, days 1 and 15; and prednisone, $80 \mathrm{mg}$, days 1-14) combined with local radiotherapy (2 Gy, days 1-20). Residual lesions were not observed on CT scans, and a BM biopsy demonstrated recovery of hematopoiesis and a disappearance of fibrosis. The patient was followed-up until relapse occurred after 2 years, and he was subsequently subjected to stem cell transplantation. Written informed consent was obtained from the patient prior to publication of this study.

\section{Discussion}

Primary or idiopathic MF is a myeloproliferative disorder characterized by myeloid metaplasia, evident splenomegaly, pancytopenia and a leukoerythroblastic peripheral blood smear. SMF occurs in a variety of systemic diseases, including tuberculosis, metastatic carcinoma, osteopetrosis and toxic marrow injury following irradiation or chemical exposure $(19,20)$. SMF is also observed in a variety of hematological malignancies, including acute megakaryoblastic leukemia, chronic myeloid leukemia and hairy cell leukemia. However, SMF is uncommon in malignant lymphoma and reported cases of MF associated with HL are extremely rare.

In the present study, at the time of NSHL diagnosis, the patient's BM aspiration was a dry tap. A BM biopsy specimen demonstrated MF representing the proliferation of fibroblasts and reticulin fiber. No apparent increase in megakaryocytes was observed. Infiltration by lymphoma cells was not confirmed in the BM biopsy specimen or in the BM aspiration smear, when it became available. The fibrosis was reversible following successful chemotherapy for HL.
To the best of our knowledge, only 7 previous reports of MF associated with HL exist (Table I) (8-11). The patient ages were variable (median, 31 years; range, 12-50 years) and no significant difference in incidence based on gender was identified (male, 4; female, 3 ). The patients, with the exception of 1 case, exhibited pancytopenia without BM invasion by the lymphoma cells; in addition, 5 patients experienced bicytopenia and 3 patients had BM invasion by lymphoma cells. Furthermore, 6 of the 7 patients presented lymph node swelling, 5 demonstrated splenomegaly and 4 had hepatomegaly. The histological type of HL was reported to be of the nodular sclerosis subtype in 3 cases, lymphocyte-depleted in 2 cases, and of mixed cellularity or lymphocyte-predominant in one case each. All the patients were treated with chemotherapy, but survival times differed widely. Patients without marrow involvement presented a relatively good prognosis (survival, 9-11 years). However, marrow involvement was common, and those patients had an extremely poor prognosis (survival, 3 months to 4 years).

No specific association is known between MF and lymphoma, despite a few reported cases of MF complicated by concomitant or subsequent lymphoma (21). However, lymphoma is a recognized cause of MF, although it is uncommon. Gisselbrecht et al (22) reported that there were no patients with MF in a series of 1,883 patients with diffuse aggressive NHL. In addition, the pathogenesis of the fibrotic change in the BM of HL patients is unknown. Fibroblasts have been reported to be stimulated by certain cytokines, such as transforming growth factor- $\beta$ (TGF- $\beta$ ), platelet-derived growth factor (PDGF) and basic fibroblast growth factor (bFGF) (23). These cytokines are known to play an important role in the development of stromal proliferation. PDGF induces the proliferation of fibroblasts, while TGF- $\beta$ induces the synthesis and accumulation of extracellular matrix proteins, including fibronectin and type I and III collagens (24). Megakaryocytes and monocytes have been reported to be sources of these cytokines. In cases of lymphoma with SMF, PDGF is expressed in monocytes (25). T cells are not known to secrete PDGF, which causes MF in myeloproliferative diseases; however, T cells secrete TGF, which can cause fibrosis. Plasma TGF- $\beta$ levels were found to be elevated in cases of MF associated with peripheral T-cell (8), cytotoxic T-cell (12) and splenic marginal zone lymphomas (13). In addition, there is evidence that the nodular sclerosis of certain cases of Hodgkin's disease is due to the increased TGF levels $(26,27)$.

In the present case, the patient developed MF and lymphoma simultaneously, and these regressed completely following chemotherapy. In conclusion, the disease status of MF was similar with that of HL, suggesting that HL plays an important role in the pathogenesis of MF. Certain cytokines are hypothesized to stimulate the growth of fibroblasts and synthesis of collagen in BM fibroblasts. Further studies and additional case reports will be required to clarify the pathogenesis of SMF and improve our understanding of the immunological dysregulation associated with lymphoma.

\section{Acknowledgements}

This study was supported by a grant from the Tianjin Cancer major special project (no. 12ZCDZSY18000). 


\section{References}

1. Younes A: Novel treatment strategies for patients with relapsed classical Hodgkin lymphoma. Hematology 2009: 507-519, 2009.

2. Eichenauer DA, Böll B and Diehl V: Pharmacotherapy of Hodgkin lymphoma: standard approaches and future perspectives. Expert Opin Pharmacother 15: 1139-1151, 2014.

3. Tefferi A: Pathogenesis of myelofibrosis with myeloid metaplasia. J Clin Oncol 23: 8520-8530, 2005.

4. Tefferi A: Primary myelofibrosis: 2014 update on diagnosis, risk-stratification, and management. Am J Hematol 89: 915-925, 2014.

5. Niino D, Tsuchiya T, Tomonaga M, Miyazaki Y and Ohshima K: Clinicopathological features of acute megakaryoblastic leukaemia: Relationship between fibrosis and platelet-derived growth factor. Pathol Int 63: 141-149, 2013.

6. Aljinovic N, Bogusz AM, Kantarci S, Buck TP and Dewar R: An unusual case of Philadelphia chromosome-positive chronic myelogenous leukemia with trisomy 19 presenting with megakaryoblastosis and myelofibrosis. Arch Pathol Lab Med 137: 1147-1151, 2013.

7. Araki H, Matsunaga T, Murase K, Kuroda H, Kuribayashi K, Terui $\mathrm{T}$ and Niitsu Y: Successful induction and complete improvement of myelofibrosis and erythema nosodum with cladribine in a case of hairy cell leukemia. Gan To Kagaku Ryoho 31: 965-969, 2004 (In Japanese).

8. Okabe S, Miyazawa K, Lguchi T, Sumi M, Takaku T, Ito Y, Kimura Y, Serizawa H, Mukai K and Ohyashiki K: Peripheral T-cell lymphoma together with myelofibrosis with elevated plasma transforming growth factor- $\beta 1$. Leuk Lymphoma 46: 599-602, 2005

9. Kikukawa M, Umahara T, Kikawada M, Kanaya K, Sakurai H, Shin K, Mori M and Iwamoto T: Peripheral T-cell lymphoma presenting as myelofibrosis with the expression of basic fibroblast growth factor. Geriatr Gerontol Int 9: 395-398, 2009.

10. Matsui K, Adachi M, Tominaga T, Shinohara S and Kamei T: Angioimmunoblastic T cell lymphoma associated with reversible myelofibrosis. Int Med 47: 1921-1924, 2008.

11. Rao SA, Gottesman SR, Nguyen MC and Braverman AS: T cell lymphoma associated with myelofibrosis. Leuk Lymphoma 44: 715-718, 2003.

12. Abe Y, Ohshima K, Shiratsuchi M, Honda K, Nishimura J, Nawata $\mathrm{H}$ and Muta K: Cytotoxic T-cell lymphoma presenting as secondary myelofibrosis with high levels of PDGF and TGF-beta. Eur J Haematol 66: 210-212, 2001.

13. Matsunaga T, Takemoto N, Miyajima N, Okuda T, Nagashima H, Sato T, Terui T, Sasaki H, Ohmi N, et al: Splenic marginal zone lymphoma presenting as myelofibrosis associated with bone marrow involvement of lymphoma cells which secrete a large amount of TGF-beta. Ann Hematol 83: 322-325, 2004.
14. Meadows LM, Rosse WR, Moore JO, Crawford J, Laszlo J and Kaufman RE: Hodgkin's disease presenting as myelofibrosis. Cancer 64: 1720-1726, 1989.

15. Carroll WL, Berberich FR and Glader BE: Pancytopenia with myelofibrosis. An unusual presentation of childhood Hodgkin's disease. Clin Pediatr (Phila) 25: 106-108, 1986.

16. Vukelja SJ, Krishnan J, Ward FT and Redmond J III: Synchronous Hodgkin's disease and myelofibrosis terminating with granulocytic sarcoma and acute megakaryocytic leukemia. South Med J 83: 1317-1320, 1990.

17. Viola MV, Kovi J and Nukhopadhyay M: Reversal of myelofibrosis in Hodgkin disease. JAMA 223: 1145-1146, 1973.

18. Izumi $\mathrm{T}$ and Ozawa $\mathrm{K}$ : Clinical staging classification of non-Hodgkin's lymphoma. Nihon Rinsho 58: 598-601, 2000 (In Japanese)

19. Viallard JF, Parrens M, Boiron JM, Texier J, Mercie P and Pellegrin JL: Reversible myelofibrosis induced by tuberculosis. Clin Infect Dis 34: 1641-1643, 2002.

20. Atasever T, Vural G, Yenidünya S, Ataoğlu O, Atavci S and Unlü M: Tc-99m MIBI bone marrow uptake in bone marrow fibrosis secondary to metastatic breast carcinoma. Clin Nucl Med 22: 655-656, 1997.

21. Gabali AM, Jazaerly T, Chang CC, Cleveland R and Kass L: Simultaneous hepatosplenic T-cell lymphoma and myelofibrosis. Avicenna J Med 4: 34-36, 2014.

22. Gisselbrecht C, Gaulard P, Lepage E, Coiffier B, Brière J, Haioun C, Cazals-Hatem D, Bosly A, Xerri L, Tilly H, et al: Prognostic significance of T-cell phenotype in aggressive non-Hodgkin's lymphomas. Groupe d'Etudes des Lymphomes de l'Adulte (GELA). Blood 92: 76-82, 1998.

23. Tefferi A: Myelofibrosis with myeloid metaplasia. N Engl J Med 342: 1255-1265, 2000.

24. Charni Chaabane S, Coomans de Brachène A, Essaghir A, Velghe A, Lo Re S, Stockis J, Lucas S, Khachigian LM, Huaux F and Demoulin JB. PDGF-D expression is down-regulated by TGF $\beta$ in fibroblasts. PLoS One 9: e108656, 2014.

25. Wang JC, Chang TH, Goldberg A, Novetsky AD, Lichter S and Lipton J: Quantitative analysis of growth factor production in the mechanism of fibrosis in agnogenic myeloid metaplasia. Exp Hematol 34: 1617-1623, 2006.

26. Kadin ME, Agnarsson BA, Ellingsworth LR and Newcom SR: Immunohistochemical evidence of a role for transforming growth factor beta in the pathogenesis of nodular sclerosing Hodgkin's disease. Am J Pathol 136: 1209-1214, 1990.

27. Newcom SR and Tagra KK: High molecular weight transforming growth factor beta is excreted in the urine in active nodular sclerosing Hodgkin's disease. Cancer Res 52: 6768-6773, 1992. 